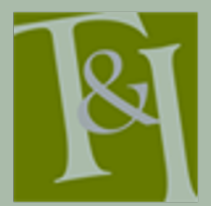

The International Journal for Translation \& Interpreting Research trans-int.org

\section{Issues in Arabic video game localization: A descriptive study}

\author{
Mohammed Al-Batineh \\ Yarmouk University, Jordan \\ m_bataineh@yu.edu.jo
}

DOI: 10.12807/ti.113202.2021.a03

\begin{abstract}
Because Arabic video game localization is a relatively new area of study, little in-depth research has been done on the topic within Arabic translation studies. The few articles that address video game localization in Arabic remain limited, either due to examining a single video game and its various localization issues, or examining various games but considering the transfer of their linguistic assets only. This paper aims to address the existing gaps in Arabic video game localization studies by delving into multiple video games localized into Arabic, and analysing the technical, linguistic, and cultural issues found within them. Technical issues are related to the Arabic script, video game variables, and subtitling conventions. Linguistic issues, on the other hand, include Arabic video game terminology and acronyms as well as the translation of proper names and NPCs' titles into Arabic. Lastly, the paper discusses cultural challenges that arise when localizing video games into Arabic, such as how to navigate nudity, profanity, and alcohol. The paper concludes by calling for more research into the area of video game localization. Such research should not only flag linguistic, cultural, and technical issues but also establish a body of literature that would help practitioners and video game developers provide a more authentic, unique gaming experience for Arab gamers.
\end{abstract}

Keywords: Localization; video games; Arabic video game localization; localization quality; localization issues.

\section{Introduction}

Due to the expansion of the Arabic video games market, several video game developers are capitalizing on this growth by providing Arabic localization of their video games. The first attempts to provide Arab gamers with localized video games came in the mid-1990s when an Arab fan, named Adnan, collaborated with Rinco (Ramar International), a Taiwanese distributor and developer of Nintendo games, on the translation of Captain Majed (1995), also known as Captain Tsubasa II (1990) on the Nintendo Entertainment System (NES) (Al-Batineh and Alawneh, in press). This initial effort is not considered an official localization attempt, as Rinco was not the official developer of the game; rather, it can be considered more of a ROM-hacking attempt, which entails the process by which gamers or fans hack the ROM data "(Read-Only Memory therefore not meant to be changed by the user) of a video game to alter various aspects of the game, including the game's language" (O'Hagan \& Mangiron, 2013, p. 10). Adnan also collaborated with Rinco to produce an Arabic translation of The Dragon (1995), which was developed by the same company. After Rinco localized video games into Arabic, no serious video localization attempts appeared until the mid-2000s when This is Football (2005) was released with an Arabic commentator in 2006. Before that, the Arab video games market relied on bootlegged copies and pirated PlayStation and PC games. 
Over the past decade, video game companies have recognized the value of the Arabic market, and thus have exponentially increased the number of video games localized into Arabic. With this increase in video games comes the increased need for localizers and Arabic localization specialists. However, in the absence of professional training for Arabic localization and the lack of scholarly studies into the practice, several linguistic and technical issues have emerged in Arabic-localized games. Video game developers have adopted a 'trial and error' method to provide Arabic localization to their games, which has resulted, in some cases, in awkward Arabic versions of said games.

Video game developers rely on outsourcing their Arabic localization needs. They provide vendors or translation agencies with the linguistic content of the video game in a text-only or spreadsheet format without adequate context about gameplay (O'Hagan \& Mangiron, 2013, p. 269). For video game companies, the localization process is concentrated on the linguistic transfer of textual assets. This narrow focus omits key steps necessary to successfully translate a game for specific markets. Localization goes beyond simply transferring the linguistic assets of the software product (Jiménez-Crespo, 2013, p. 12; 2019, p. 299). It requires much more than "text-to-text translation" (Bernal-Merino, 2020, p. 298) and must take into consideration the sociocultural, religious characteristics of the target market and accordingly adapt the game for the target culture. In this regard, Mangiron (2016) argues that video game localization consists of "making a game suitable to be sold in other territories, and it involves complex technical, linguistic, cultural, legal and marketing processes" (p. 190). These processes also include matching the layout, text-orientation, and language script requirements and conventions to those of the target language. This way, the target video game has the "feel and look" of an original game (LISA, 2004, p. 11).

A successful localization of any software product, be it a computer program, website or a video game, relies on a continual process known as GILT (Globalization, Internationalization, Localization and Translation) (JiménezCrespo, 2013, p. 11). Localization is "one of a number of interdependent processes and cannot be fully (or correctly) understood without being contextualized in reference to them" (Dunne, 2006, p. 4). In this context, localization depends on the internationalization stage, which entails preparing software products in a way that facilitates localization. Such processes could include developing the program or video game code to technically support different languages, creating a culture-neutral content, or eliminating graphics that could cause issues in target cultures. The successful implementation of the internationalization strategy facilitates localization and eliminates costly problems before localization even begins (Dunne, 2006, p. 6).

Video game localization not only involves the translation of in-game texts but also includes language encoding, text orientation, texts on images, subtitling, and dubbing. After configuring the assets for the target market, the localization changes must then be applied within the video game. Even if the translators provide an excellent rendition of the video game's textual assets, applying the translation without any technical issues is not guaranteed. This issue becomes more serious when dealing with Arabic. Arabic text orientation, cursive script, and encoding can be difficult and sometimes impossible to apply when localizing a specific game. In this regard, several video game localization scholars have discussed technical, linguistic and cultural issues in video game localization generally (Bernal-Merino, 2007, 2015; Czech, 2013; Mangiron, 2018; O'Hagan \& Mangiron, 2013), but little attention has been paid specifically to this activity in Arabic.

The present paper is descriptive in nature and provides an overview of the technical, linguistic, and cultural issues in Arabic video game localization. The technical issues discussed in this paper are related to text directionality, cursive script, and orientation as well as the translation of variables in video game 
strings and subtitling conventions. Linguistic issues, on the other hand, include Arabic video game terminology, the translation of proper names and titles of non-player characters (NPCs), and the assets left untranslated when video games are localized into Arabic. The last section discusses the cultural challenges, such as nudity and profanity, that are faced when producing an Arabic video game localization. The discussion in the following section relies on data collected from Arabic localized video games on different platforms such as PlayStation 4 and Xbox One.

\section{Literature review}

Several video game scholars have highlighted the technical, linguistic, and cultural issues that arise when localizing video games into other languages. For instance, Bernal-Merino (2007) discusses the different textual assets found in video games (the manual, packaging, "readme" file, official website, dialogue for dubbing or subtitling, user interface [UI], graphics with words) and how these textual types could be challenging for translators and localizers. He points out that a main challenge in working with video game assets is that they are provided to the translators in a spreadsheet format without adequate contextual information. Another issue is the non-linearity of gameplay and how it causes the video game's linguistic content to be fragmented. Bernal-Merino (2007) explains that in video games, "most things happen as and when players trigger them through their actions" (p.5). This means that textual data like dialogue appears in gameplay based on the player's actions within the game, as opposed to an easier-to-follow chronological order. It also means that the information in the spreadsheets provided to localizers is often fragmented, and the textual elements "might not have any chronological relevance to each other" (BernalMerino 2007, p. 5).

Bernal-Merino (2007) also highlights another challenge that video game translators face: the use of variables by game developers. Such variables are sometimes found in the translatable strings and could include name, gender, nationality, and others. He gives the following examples to illustrate his point: " $/ \mathrm{n}$ nameofnation $/ \mathrm{n}$ is attacking you!". This string contains translatable and non-translatable elements (variable). The " $/ \mathrm{n}$ nameofnation $/ \mathrm{n}$ " is a variable that changes depending on the "name" of the nation that is attacking the player. In such a case, "translators have to be aware of which strings belong to the game code and which strings belong to localizable assets" (Bernal-Merino, 2007, p. 6). In addition to variables in video game strings, some languages are synthetic languages, i.e., languages that depend on inflections to build and express syntactic relations. In such cases, the grammatical gender and number of the variable should be matched with other grammatical elements in the string to avoid awkward translations. This issue is also highlighted in Bernal-Merino's (2020) latest article on game localization quality. He points out that English "syntax and morphology allow for easy sentence building" (p. 305) using variables; however, "due to the existence of masculine, feminine and neutral linguistic gender and the way in which articles and adjectives are declined in agreement with nouns" (Bernal-Merino, 2020, p. 305), other languages such as Spanish or German cannot be made to fit video game strings with variables that were originally developed to build sentences in English.

Czech (2013) also touches upon some of the issues in video game localization, and discusses the linguistic, technical, and culture-specific challenges in the localization of Call of Duty $(2009,2010,2011)$ into Polish. Czech (2013) reports several linguistic issues within the Polish localization of the game, the most important being the translation of internal video game terminology. This, he argues, is a "result of misinterpretation caused by insufficient contextual information" (Czech, 2013, p. 14). Without adequate 
context, a translator may not know the best way to translate a word with multiple meanings. For example, with the word "customs", a mistranslation may occur since the word can mean customs in an airport or customs related to a specific culture. He also discusses hard-coding and string automatization in video games as being problematic in the localization process. As previously mentioned, some video game localization strings include translatable and untranslatable variables. The translatable variables adhere to their syntactic type, gender, and number, and may cause major issues when a game is localized into a language with a quite different syntactic and morphological system. Grammatical problems become inevitable in such contexts due to string automatization, which generates word strings according to the player's moves and the choices they take in the game. Czech (2013) also notes another issue in the Polish context - one that is not directly related to the localization of actual games, but rather to the mindset of the gamers. Czech (2013) points out that Polish gamers were "accustomed to very scarce localizing or no localizing at all", which led them to absorb the English game terminology they were exposed to (Czech, 2013, p. 20). This created something of a norm in the gaming communities that involved using borrowed video game terminology. Developers, on the other hand, insisted that the borrowings be properly translated into Polish. This debate presents Polish video game localizers with further challenges as they face an "irresolvable problem of dealing with such lexical items" (Czech, 2013, p. 20).

Other scholars have analysed the localization of video game linguistic and textual assets into Baltic languages. For instance, Šiaučiūnè and Liubinienè (2011) adopt a linguistic comparative analysis to evaluate the Lithuanian localization of Magic Encyclopedia: the First Story (2008). The researchers analysed a corpus of 533 words including terms, menu items, simple and complex sentences. They found that the translation of the games' textual assets was mostly literal and source oriented. Šiaučiūnè and Liubinienè (2011) report multiple issues in the Lithuanian translation including mistranslations, spelling typos, and stylistic issues, all having a negative impact on the game's quality and playability. They pointed out that the lack of training programs and professional video game localizers is a main reason for low-quality localized video games in Lithuanian.

On the other hand, some researchers have investigated the localization of textual assets of video games into English. For instance, Sharifi (2016) discusses the translation and localization of Garshasp: Temple of the Dragon (2012), the first Persian video game to be localized into English. To this end, the researcher focuses on the in-game texts and how translators rendered the textual assets into English. Sharifi (2016) relies on Vinay and Darbelnet's model (1995) of translation methods to provide a qualitative and quantitative descriptive analysis of the data. Sharifi (2016) reports that the most dominant translation strategies were modulation and deletion, as well as addition and literal translation.

Similarly, Mangiron (2021) investigates the localization of Japanese video games into English. She analyzes three localization case studies, Persona (1996-present), Phoenix Wright: Ace Attorney (2005-present), and Yakuza (2005-present), to reveal how the localization of Japanese video games evolved over time due to gamers' input into and criticism of the localization. Mangiron (2021) adopts a participant-oriented approach, focusing on the agents (such as translators, editors, producers) involved in the localization process, and how the end-users received the localization of the selected video games. She also employs a documentary approach with data collected from online interviews, blogs, specialized websites, and discussion forums that details the opinions of localization practitioners, game critics, and players about the localization of the chosen three games (Mangiron, 2021, p. 3). Mangiron's (2021) study reveals remarkable findings on the way western gamers would like to have Japanese games localized. The study indicates that gamers prefer to play Japanese video 
games that maintain the foreign flavour of the source culture. She argues that the gamers' expectations of the localization of Japanese video games into English have shifted game developers' localization strategies "from a higher to a lower degree of cultural adaptation in order to meet target markets' expectations" (Mangiron, 2021, p. 1). This strategy has helped Japanese video game companies widen their fan base. She argues that "gamers are very active and vocal on Internet forums" and "paying attention to them can help identify the most suitable localization approach" (Mangiron, 2021, p. 14).

Video game localization in the Arabic context has attracted the attention of a limited number of scholars. Such studies were conducted as either an MA thesis (Abu Kishek, 2016) or a PhD dissertation (Al-Mazrooa, 2018). In her MA thesis, Abu Kishek (2016) analyses the Arabic translation of textual assets in Assassin's Creed: Syndicate (2015) and Tomb Raider (2013), including ingame terminology, on-screen messages, and subtitles. She reports that the Tomb Raider (2013) translator succeeded in rendering several menu items into Arabic but failed in others. For example, some menu items were left in English or literally translated into Arabic, thus producing an ambiguous and misleading translation. Similar linguistic issues were also found in the Arabic version of textual assets in Assassin's Creed: Syndicate (2015). Abu Kishek (2016) also discusses the Arabic subtitles in both games. She provides examples of successful idiomatic translation in each video game, highlighting instances where Arabic subtitles were domesticated. For instance, the word "pig" in the English version was replaced with "sheep". In another instance, the translator replaced "Christ" with "heaven" in Arabic. Abu Kishek (2016) argues that the translator succeeded in domesticating such cultural items as they are more appealing and closer to the Arab culture. Additionally, Abu Kishek (2016) points out that the orientation of the Arabic-language user interface (UI) in Assassin's Creed: Syndicate (2015) and Tomb Raider (2013) does not follow the Arabic right-to-left text convention.

Another important study on Arabic video game localization was conducted by Al-Mazrooa (2018) in her PhD thesis; she devoted an entire section to reporting the results of a case study on the Arabic localization of FIFA 15 (2014). This case study provides a detailed analysis of the game's three Arabic localization assets: the 'box and docs' (game packaging), in-game text, and audio assets. In her analysis of the Arabic game's packaging, Al-Mazrooa (2018) remarks that the front cover includes both Arabic and English information related to the game, such as console and age rating. She notes that brand names on the front cover-such as EA Sports and PlayStation-were left in English. She also reports that the Arabic version's front cover displayed the European age rating information only, arguing that "a possible reason for using a European rating system for Arabic localized video games is the absence of an Arabic rating system recognized by all or most Arabic countries" (Al-Mazrooa, 2018, p. 47). The back cover, on the other hand, mixes Arabic and English; only promotional texts on the back cover and the information textbox are provided in Arabic (Al-Mazrooa, 2018, p. 54). Furthermore, she reports that the Arabic text on the back cover is not right aligned, which is an important aspect of localizing into Arabic. Al-Mazrooa (2018) also discusses the quality of the Arabic box-and-docs translation and points out that the translation includes several "misleading translations" and omissions (p. 56).

Al-Mazrooa (2018) additionally discusses the linguistic transfer of the user interface (UI) items into Arabic by analysing the quality of the Arabic translation. She finds that terminology inconsistency was a serious issue in the Arabic version where two or more Arabic terms are used to refer to the same English source term. Al-Mazrooa (2018) elaborates that sometimes a specific term has different meanings depending on its place in the menu. In such cases, adopting one consistent translation for this specific term is problematic. She notes that localizers are faced with rendering linguistic items and strings into 
Arabic using only the fragmented, out-of-order references in a spreadsheet. This decontextualized list of strings prevents translators from identifying the correct denotative meaning of UI items (Al-Mazrooa, 2018, p. 73).

Al-Mazrooa (2018) then examines an important element of Arabic video game localization: UI directionality. In Arabic video games the UI should be right-aligned, 'mirroring' the English version of the game. Mirroring, in this context, refers to the "process of creating an inverted UI design in order for RTL texts to display naturally, starting from the right margin" (Al-Mazrooa, 2018, p. 77). In this regard, she points out that:

The Arabic translation of the FIFA 15 EA Sports video game UI does not constantly respect the Arabic language directionality. Some UI elements appear from right to left (RTL), achieving technical domestication, while others disorient Arabic text presentation, creating technical foreignisation (Al-Mazrooa, 2018, p. 76).

Technical foreignization is common in Arabic software and video game localization. The proper RTL rendering of software, such as the orientation of the menus and the layout, has rarely been adapted into Arabic.

The last localization asset discussed by Al-Mazrooa (2018) is voiceover. Four types of voiced presentations were used in FIFA 2015 (2014), including sideline commentator, career-mode radio announcer, commentary, and public announcer (Al-Mazrooa, 2018, p. 80). Al-Mazrooa (2018) reports that only the commentary is re-voiced in Arabic, which uses the voice of Esam El-Sha'waly, a famous Tunisian-born football commentator in the Arab World. The researcher also argues that the Arabic commentary is "natural and reflects real characteristics of the Arabic football commentary" (p. 96), which provides Arab gamers with an enjoyable gaming experience.

Following in the steps of Abu Kishek (2016), Mahasneh and Abu Kishek (2018) qualitatively reinvestigate and analyse the Arabic localized version of Tomb Raider (2013). The researchers collected their data from the in-game texts, including the Arabic UI and subtitles of the cutscenes (non-interactive scenes) and voiceovers. They find that many user interface items included in the main menu of the game were translated literally into Arabic, ignoring their communicative aspect. They argue that several menu item translations were detached from their context, which consequently impacted the quality of their translation and their communicative purpose. Al-Mazrooa (2018) reported a similar issue in the localization of FIFA 15 into Arabic. Mahasneh and Abu Kishek (2018) state that the Arabic version of Tomb Raider (2013) succeeded by adopting functional target-oriented translation to render some menu items, especially those related to weapons. They further highlight the importance of video game acronyms and their rendering into Arabic. Mahasneh and Abu Kishek (2018) reported that the Arabic version of the game retained acronyms in English, impacting its accessibility for gamers who do not read that language.

Mahasneh and Abu Kishek's analysis (2018) of Arabic subtitles also focused on the transfer of cultural taboos, idioms, and metaphoric language. They report that taboo words related to profanity and religion were toned down in the Arabic version of the game. Idioms and metaphorical expressions were rendered into Arabic by providing a functional equivalent, which resulted in a domesticated translation. Mahasneh and Abu Kishek's (2018) study focused solely on the translation quality of Tomb Raider (2013) into Arabic; however, technical aspects of video game localization were not discussed. These aspects constitute a serious challenge to Arabic video game localization, which entails best practices for localizing text orientation, Arabic font, and script directionality.

The present study provides a more comprehensive overview of the technical, linguistic, and cultural issues of Arabic video game localization. It adopted a qualitative, descriptive approach to exploring localization issues 
found in twenty-eight video games officially localized into Arabic (See Appendix 1). The video games were either played by the researcher himself or criticized for localization issues by the Arab gamer community. In the latter case, the researcher installed the video game on his personal console and navigated through the game to ensure that the issue was present. The technical issues, discussed below, are related to Arabic script (such as directionality, cursive script and text orientation), variables, and subtitling conventions. The linguistic issues, on the other hand, include Arabic video game terminology and acronyms, the translation of proper names and titles of NPCs into Arabic, and the assets left untranslated in the Arabic version. The last section discusses cultural challenges found in Arabic video game localization such as games featuring nudity and profanity.

\section{Findings and discussion}

\subsection{Technical issues}

\subsubsection{Directionality}

Most often, Arabic is written and read from right to left; however, in certain contexts, some parts inside a sentence are written and read from left to right. Such cases include sentences with numbers and Latin texts, which are common in video games. This aspect makes Arabic a bi-directional (BiDi) language, and Arabic texts in any localized software should also reflect this characteristic. Several video games do not support BiDi, thereby posing difficulties for their corresponding Arabic versions. This complication becomes serious when numbers, Latin words, and certain signs are found in one line or sentence. The following example is taken from PlayerUnknown's Battlegrounds (2017) on Xbox One. When the player dies, a message pops up saying " $[\mathrm{X}]$ player killed with [X weapon]", where the screen name of the player and weapon type are presented in English inside the Arabic sentence.

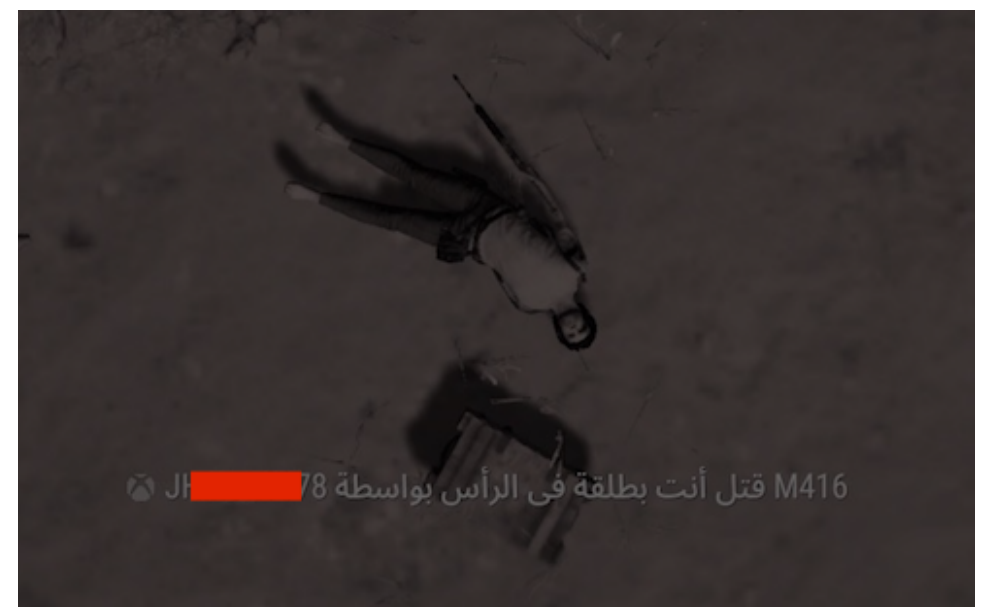

Figure 1. Incorrect display of Arabic BiDi text taken from PlayerUnknown's Battlegrounds (2017) on Xbox One

The message in Figure 1 reads (my translation) "M416 [weapon] killed

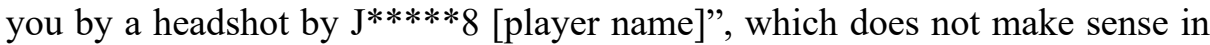
Arabic. As discussed above, the internationalization stage of the GILT process involves preparing the video-game source code to facilitate and support localization. It can be understood as the elimination or modification of problematic elements in the game upstream, including technical or cultural issues (O'Hagan \& Chandler, 2016, p. 312). The Arabic version of the game clearly does not support BiDi to accommodate Arabic RTL text orientation. 
Here at least it seems that the game code was not prepared or designed (internationalized) in a way that supports the translation/localisation task in Arabic. A careful internationalization strategy would accommodate for the technical peculiarities of the target languages and thus prevent potential technical issues in the localized version.

\subsubsection{Cursive script}

Arabic is written in a cursive style, which means that letters are always connected, and this style should be supported by the game code or game engine to improve Arabic gameplay. Such a system would ensure that the in-game Arabic script is displayed correctly with all letters connected and read from right to left. Some localized games, from well-known developers such as KONAMI, do not support Arabic cursive script, making it difficult for Arab gamers to read certain menu items.

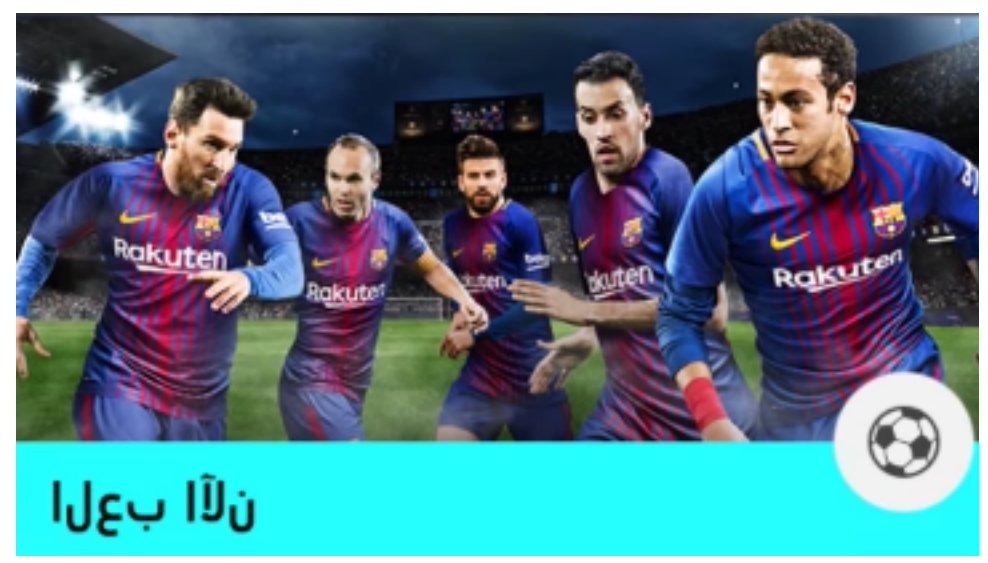

Figure 2. Disconnected Arabic letter in PES 2018 (2017)

In KONAMI's Pro Evolution Soccer (PES) 2018 (2017), some menu items do not support Arabic cursive script. As seen in Figure 2, it is difficult to read the Arabic "العب الان" (Play Now) because the letters are disconnected and displayed from left to right. However, other menu items appear correctly. Cursive script issues in the Arabic video game were solved post-launch, which cost KONAMI more money, effort, and time. It is worth mentioning here that fixing localization issues once a game is on the market depends on the return on investment (ROI) in doing so: that is, measuring the cost of fixing the localization against the potential increased profit those fixes will generate from the target market. If the profit gains exceed the localization repair costs, the company will, in most cases, fix the problem. The PES (1995-present) series is very popular in the Arab world, and an investment in correcting localization issues in the Arabic version will likely yield a positive return.

In other similar cases, the game developer does not fix disconnected Arabic, which could be due to weak sales in the target market. The Arabic localization of Goat Simulator (2015) is a case in point. All menu items and ingame text display disconnected Arabic letters (See Figure 3-A). 


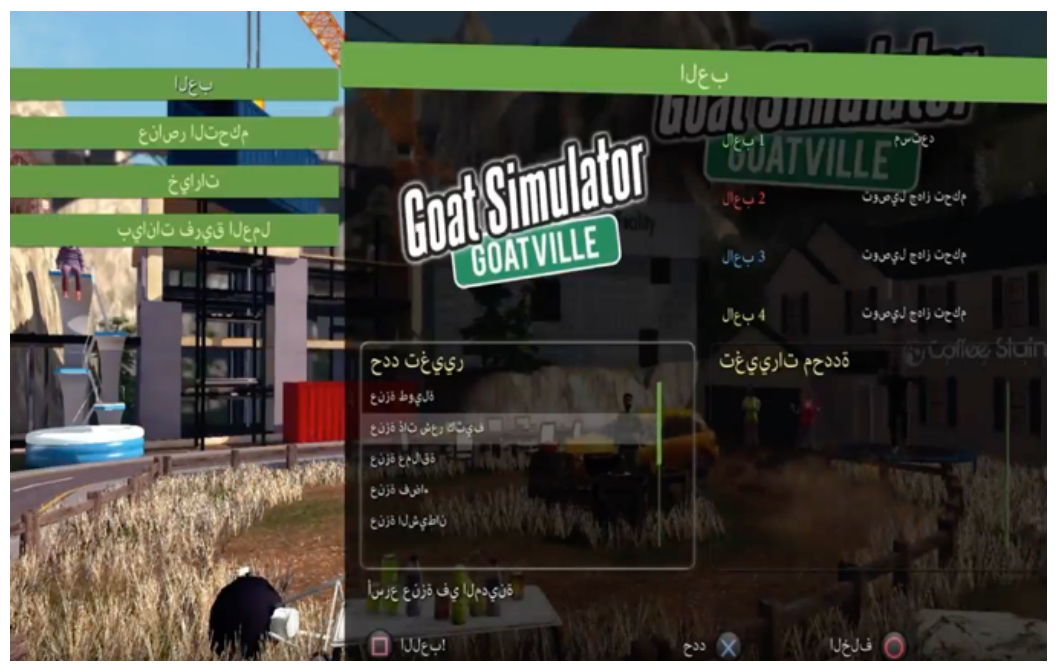

Figure 3-A. Disconnected Arabic letters in Goat Simulator (2015)

Due to menu items with either disconnected letters or disconnected letters and wrong text directionality, Arab gamers would have difficulty reading ingame texts. Recently, Arab ROM-hackers fixed Goat Simulator (2015) and made it available with the correct script and layout (See Figure 3-B).

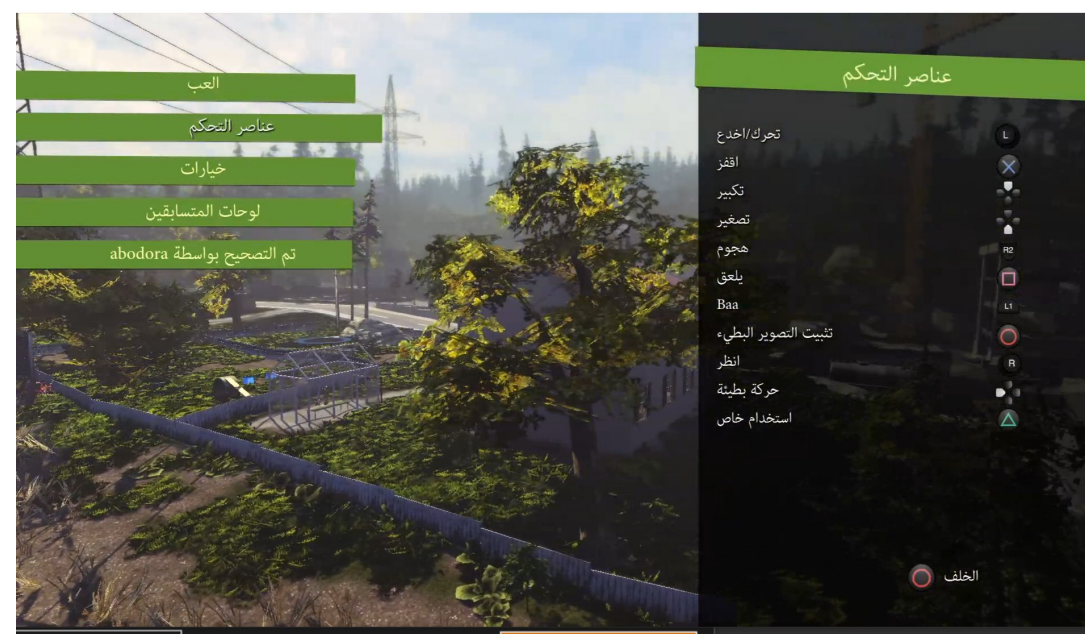

Figure 3-B. Goat Simulator (2015) after Arab ROM-hackers fixed the text

This issue of disconnected Arabic letters becomes more serious and disruptive when the video game includes subtitles for the voiceover. Recently, Graceful Decay's Maquette (2021) was released with disconnected Arabic script in the main menu and the subtitles (See figures 4-A and 4-B).

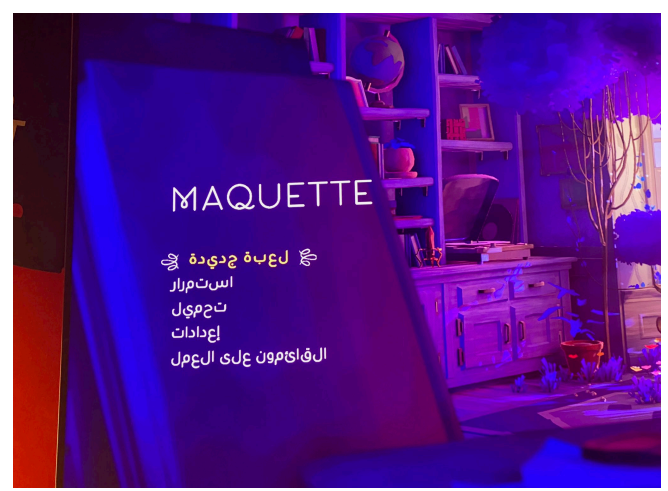

Figure 4-A. Disconnected Arabic letters in the Maquette (2021) main menu 


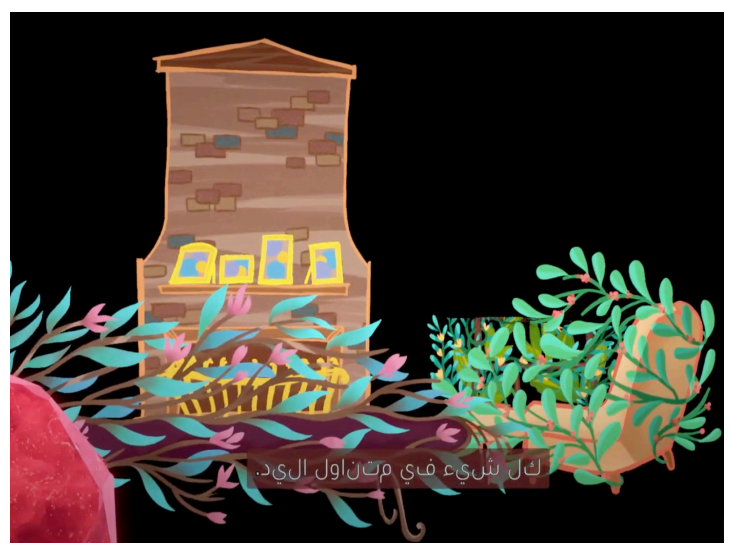

Figure 4-B. Disconnected Arabic letters in the Maquette (2021) subtitles

Such issues negatively impact video game sales in the target market and also hinder playability, which is, as Bernal-Merino (2020, p. 299) states, a key quality metric of video game localization.

\subsubsection{Text orientation and UI layout}

English or western games are designed in order to accommodate left-to-right languages. In the case of an Arabic game, players would expect right-to-left text orientation and interface layout. Thus, localizing game menus should not only focus on the linguistic transfer of the UI's lexical items but also cater for the display expectations of an Arabic UI. As Yannick Theler, the Managing Director of Ubisoft Abu Dhabi studio, points out:

[w] hat we see as a Westerner with our eyes, how we look at the computer when we play a game, it's not exactly the way they look at them here [in the Arab World]. For me I look at the left side to find a certain button. They'll [Arab gamers] tend to look to the right side (Campbell, 2013).

Almost all video games (with a few exceptions) localized into Arabic ignore this fact.

A UI layout also includes text alignment, and in Arabic specifically the ingame text should be right-aligned. The recent video game Ghost of Tsushima (2020) includes Arabic localization; however, the game does not support Arabic RTL orientation or UI layout. Consider figures 5-A and 5-B.

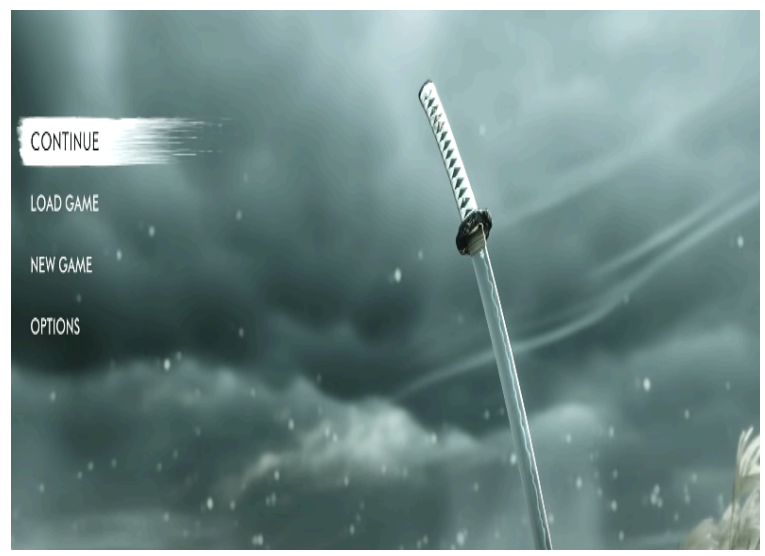

Figure 5-A. Original English version of Ghost of Tsushima (2020) 
In Figure 5-A above, the English menu items are aligned to the left. However, Figure 5-B below shows this alignment is duplicated for the Arabic version's layout and text alignment.

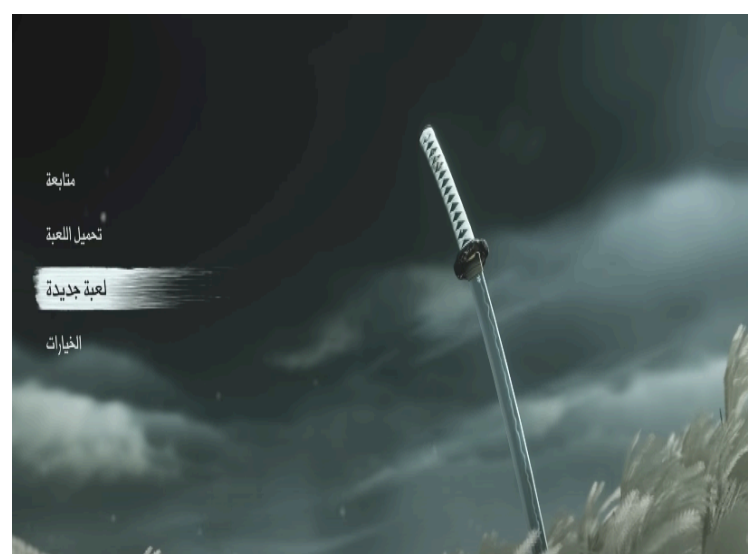

Figure 5-B. Localized Arabic version of Ghost of Tsushima (2020)

When localizing games into Arabic, the entire game layout, including the main menu, should be mirrored on the right side; this key step of localization should be considered during the internationalization process of video game development. Such adjustments in game UI and layout are costly and require more time and effort from the video game developer. Thus, some developers opt for "fit-for-purpose" localization, where the main aim would be functionality with a focus on the transfer of the linguistic content into the target language. That is, if the video game is functional and playable in the target language, other layout and cosmetic issues can be ignored, especially if fixing them requires extensive time, money, and effort.

Recently, Turn 20 Studios created a successful Arabic layout in their video game Forza 6 (2015). Figure 6-A shows the English menu of the game with the typical English layout, aligning all the menu items on the left.

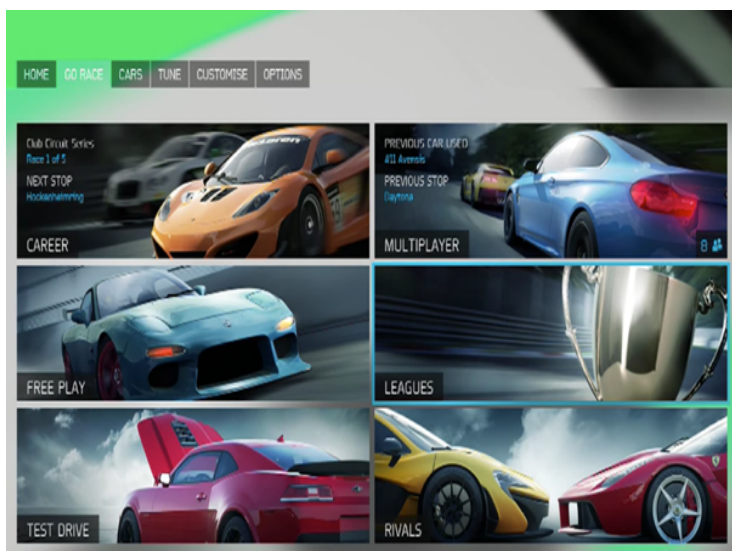

Figure 6-A. Forza 6 (2015) English menu 


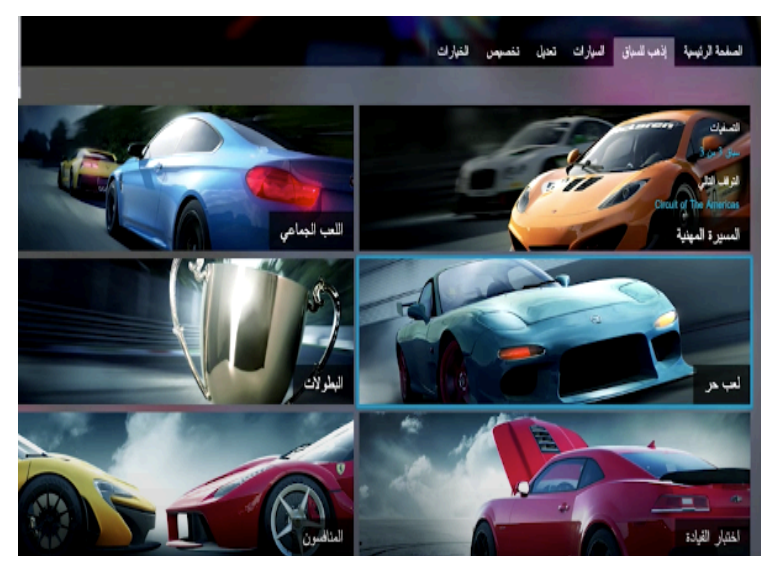

Figure 6-B. Forza 6 (2015) Arabic mirrored menu

The Arabic version of Forza 6 (2015) provides an excellent example of how video games should be mirrored when localized into Arabic (Figure 6-B). As can be seen in Figure 6-B, the whole interface and texts are right-aligned, which makes the game look and feel Arabic.

\subsubsection{Variables and Arabic localization}

Variables constitute a serious challenge in Arabic video game localization as they correlate to different values inside the game, such as the character's name or level, that could be treated differently depending on each language's syntax (See O'Hagan \& Mangiron, 2013, 132). "Variables can be used in complex ways to enhance players' immersion" by directly addressing the gamer, their chosen profile, and their performance (Bernal-Merino, 2007, p. 6). The syntactic differences between Arabic and English make it hard to provide a localized version of video game strings that contain variables. Consider the following example

Table 1. Number as a variable in Arabic and English strings

\begin{tabular}{|c|c|}
\hline \multicolumn{2}{|c|}{ You scored <number> points. } \\
\hline You scored 1 point. & لقد أحرزت ا نقطة. \\
\hline You scored 2 points. & لقد أحرزت نقطتان. \\
\hline You scored 3 points. & لقد أحرزت ب نقاط. \\
\hline You scored 4 points. & لقد أحرزت ع نقاط. \\
\hline You scored 11 points. & لقد أحرزت ال نقطة. \\
\hline You scored 1274 points. & لقد أحرزت عV I ا نقطة. \\
\hline
\end{tabular}

In Arabic, numbers have three forms: singular (1), dual (2), and plural (310). Numbers should agree with the noun they modify. Numbers above 10 must be followed by the "singular" form of the noun they modify. This system would pose a challenge for localizers as the source code of a game considers only singular or plural values, which would result in an unnatural Arabic translation of specific strings.

Because Arabic uses grammatical gender, localizing strings containing gendered variables into Arabic can be problematic. Verbs in English are genderneutral while in Arabic verbs are conjugated to reflect gender. If a translatable string contains a variable that includes the character's name, the Arabic translation of this string may not be correct as the subject must match its gendered verb. In such a case, using a male or a female form of a verb would cause problems if the string is not re-coded for both possibilities. Consider the following string: 
Table 2. Female and male character names as variables in Arabic and English strings

\begin{tabular}{|c|c|c|}
\hline String: & \multicolumn{2}{|c|}{$<$ character name> killed you in the last battle. } \\
\hline Language & English Sentence & Arabic Translation \\
\hline Female character & Lara killed you in the last battle. & لار ا قتلنك في المعركة الأخيرة. \\
\hline Male character & Jack killed you in the last battle. & جاك قتلك في المعركة الأخيرة. \\
\hline
\end{tabular}

In the hypothetical example above, the English variable $<$ character name $>$ could be replaced with Lara or Jack, with the verb form in the phrase that follows ("killed you in the last battle") remaining the same in both cases. However, this does not apply to the translation, as the Arabic equivalent of the verb "killed" must match the gender of the subject - that is, stilï for Lara and Stlï for Jack. Bernal-Merino (2020) discusses this issue and points out that such variables are problematic in languages that have masculine, feminine, and neutral linguistic genders (305). A thorough internationalization strategy would help solve such issues upfront.

\subsubsection{Subtitling conventions}

The lack of subtitling conventions is not only an issue in video game localization in Arabic, but in other languages also. However, the nature of the Arabic language makes this lack of conventions even more critical. Arabic does not include capital letters to denote proper names, but occasionally uses punctuation marks to compensate. To distinguish proper names from other words, Arabic translators may typically opt for brackets or inverted commas. Various video games have employed this practice. For instance, proper names in The Witcher 3 (2015) are enclosed in parentheses (see 1 in Table 3 below), while in The Division 2 (2018) inverted commas are used. In other video games like FIFA 2018 (2017), proper names are left in the source language, which is especially problematic for gamers who do not speak or read foreign languages.

Table 3. Proper names in subtitles from selected video games localized into Arabic

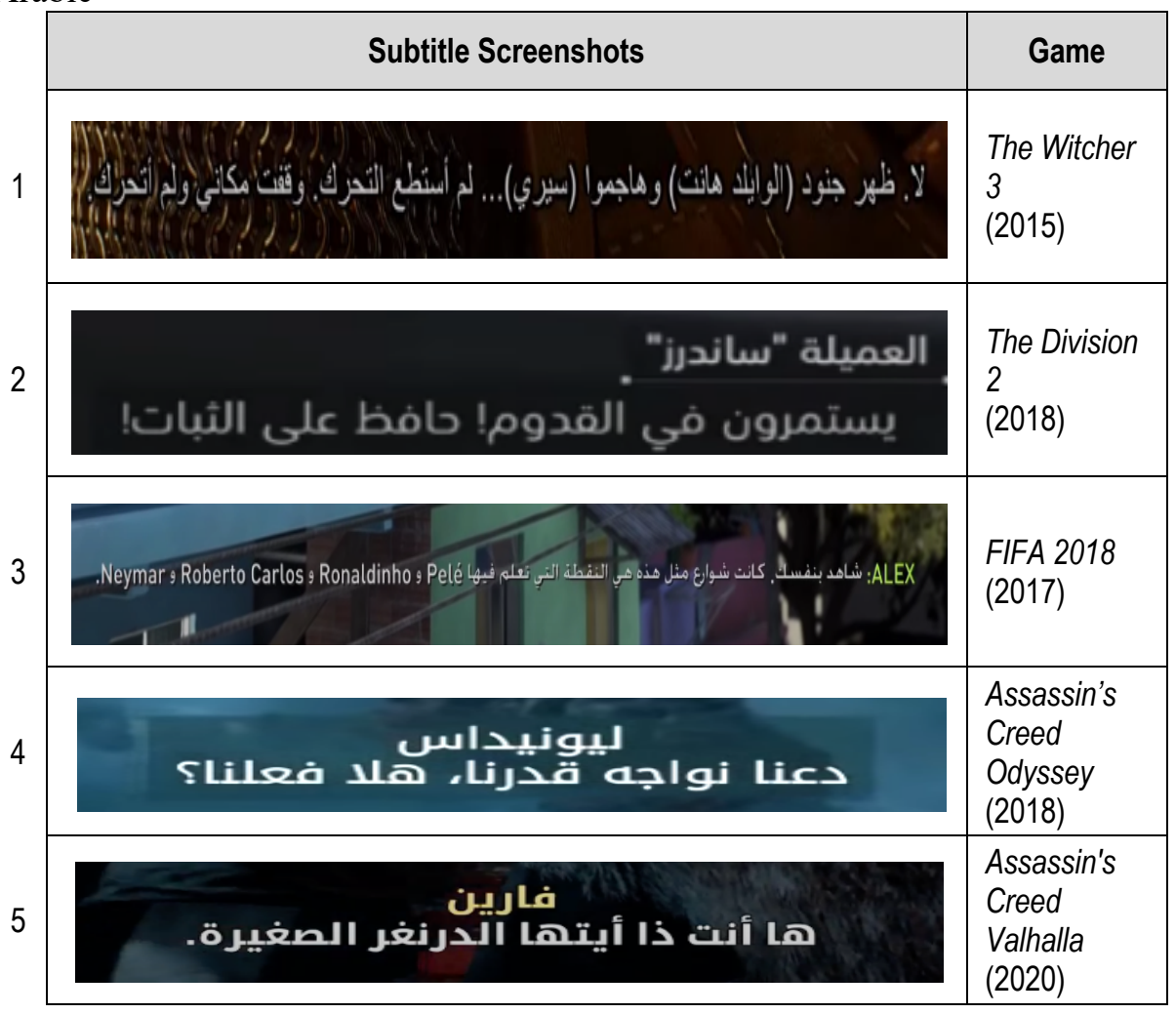


Some Arab translators and localizers believe that using punctuation to distinguish proper nouns from other words in the sentence is awkward and thus do not use them (Aziz, 1983, p. 78). This is evident in several localized video games such as Assassin's Creed Odyssey (2018), LEGO Super-Villains (2018), and Ghost of Tsushima (2020). However, trying to read transliterated proper names and distinguish them from other words can occasionally be difficult since some transliterated words look like Arabic verbs or adjectives. Using punctuation with transliterated proper names can improve readability of audiovisual texts generally and reduce confusion - indeed almost all established and common TV shows or movies with Arabic subtitles use devices such as quotation marks and/or brackets to enclose proper names (See Al-Adwan (2019)). The same practice and its advantages would apply equally to in-game texts as well.

\subsection{Linguistic issues}

\subsubsection{Proper nouns in Arabic video games}

Another critical issue in Arabic video game localizing is the translation and transliteration of proper nouns (names of persons or places). This problem stems from the fact that there are different ways to transliterate an English proper noun into Arabic. For example, in some cases, translators replace the letters of the source language word with the same letters in Arabic. In other cases, translators replace the sounds of the source language word with sounds in Arabic, or provide a translation for the proper noun. The lack of a standardized method for rendering proper names into Arabic has resulted in inconstant transliterations of proper nouns appearing in different types of translated material, including video games, movies, TV shows, and literary works. For example, one city, Skellige, in The Witcher 3 (2015) has been transliterated in three different ways:

Table 4. Arabic rendering of 'Skellige' in the Arabic version of The Witcher 3 (2015)

\begin{tabular}{|c|l|l|}
\hline English Proper Name & Arabic Transliteration & Method of Transliteration \\
\hline \multirow{5}{*}{ Skellige (Place) } & سكيلاج| & $\begin{array}{l}\text { Replace the letters of the } \\
\text { source language with letters } \\
\text { in Arabic }\end{array}$ \\
\cline { 2 - 4 } & سكيلاج & $\begin{array}{l}\text { Replace the sounds of the } \\
\text { source language with sounds } \\
\text { in Arabic }\end{array}$ \\
\cline { 2 - 4 } & $\begin{array}{l}\text { Replace the letters of the } \\
\text { source language with letters } \\
\text { in Arabic. The last letter is ₹ } \\
\text { is used in some Arabic } \\
\text { dialects in Egypt and Iraq. It } \\
\text { is not part of the Arabic } \\
\text { alphabet. }\end{array}$ \\
\hline
\end{tabular}

This issue becomes significant in video games when the gamer is faced with three different transliterations on the screen at the same time: one for his/her location on the game world map, one listing a specific quest in that location, and one in the voiceover subtitles along the bottom of the screen. Unfortunately, there is no standardized way to transliterate foreign names in translation and localization projects. However, in some localizations, the Arabic transliteration of proper nouns is managed during terminology development, which helps ensure the consistent use of a specific transliteration throughout the project. 


\subsubsection{Translating NPC titles into Arabic}

Non-player characters (NPCs) are characters inside the game that are controlled by the computer. Some NPCs - perhaps soldiers in a marketplace or monsters along a deserted road - are added to video games as a way to create a specific atmosphere or gameplay environment. NPCs are usually given a nametag that appears over the character's head. When localizers receive a localization toolkit, often in spreadsheet format, they translate NPCs into Arabic out of context, as they do not have access to images of the NPC inside the game. The differences between Arabic and English further complicate the translation of NPC details. Some NPC occupations are gender-neutral in English, while they are not so in Arabic. For instance, The Witcher 3 (2015) includes several NPCs throughout the game, such as farmer, peasant, artist, and captive. These titles are not gender-neutral in Arabic, taking different forms depending on whether they refer to a man or to a woman (See Table 5).

Table 5. Translation of Selected NPC titles from The Witcher 3 (2015)

\begin{tabular}{|l|c|}
\hline $\begin{array}{l}\text { NPC Title and Gender in English } \\
\text { Game }\end{array}$ & $\begin{array}{l}\text { NPC Title and Gender in the Arabic } \\
\text { Version }\end{array}$ \\
\hline Peasant (female NPC) & (أسير (Masculine) \\
\hline Captive (female NPC) & (Masculine) \\
\hline Artist (female NPC) & (Masculine) \\
\hline Leader (female NPC) & (Ma) \\
\hline
\end{tabular}

When rendered into Arabic, the above titles have been gendered as male, resulting in awkward translations when these titles appear above a female NPC (See figures 7-A and 7-B).

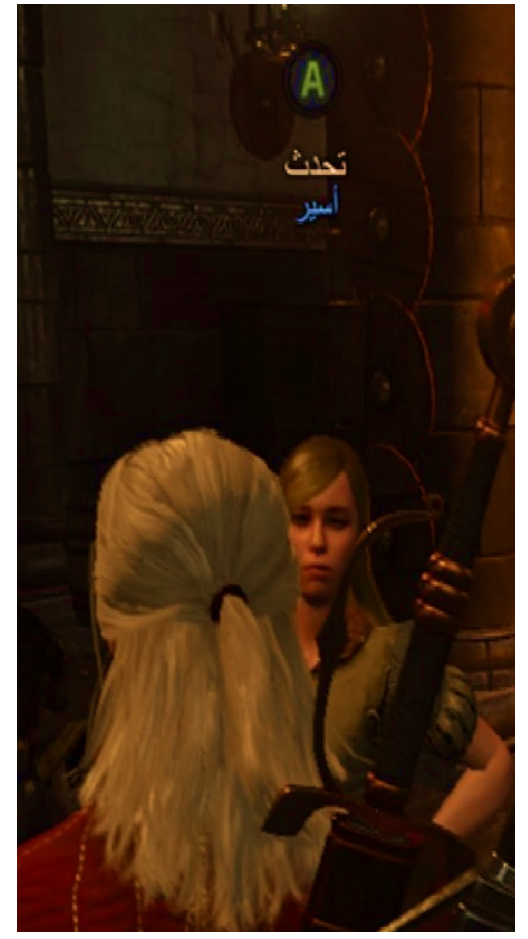

Figure 7-A. Screenshot of NPC from the Arabic version of The Witcher 3 (2015)

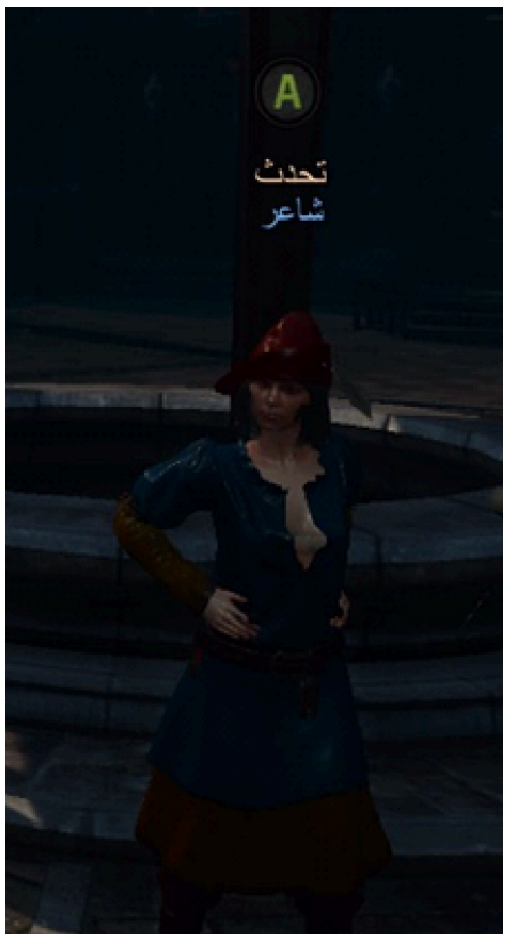

Figure 7-B. Screenshot of NPC from the Arabic version of The Witcher 3 (2015)

\subsubsection{Video game assets left in source language}

Video games include what are called 'localization assets', i.e. the elements in the video game that are localizable. These include packaging, user interface, cinematics, voiceover, and dialogue. Video game localization is delineated into 
multiple levels defined by the assets to be localized. The localization level for a given game is usually determined by marketing strategies, market size, and localization budget (O’Hagan \& Mangiron, 2013, p. 141). In this regard, three localization levels can be identified: 'box and docs localization', 'partial localization', and 'full localization' (Chandler \& Deming, 2012, pp. 9-10). Box and docs localization refers to translating the video game packaging and instructions that come with the game box. The second level, partial localization, involves localizing the "box and docs texts, as well as all the in-game text [such as user interface and menu], but maintaining audio and cinematic assets in the language of the original game, not revoicing them, and only providing subtitles", while full localization "implies translating all the game assets, including the revoicing into the target language" (Mangiron, 2021, p. 5) .

In the Arabic video game market, some developers have adopted problematic partial localization practices in which certain game assets are left in the source language. These practices negatively impact the playability of the video game. For example, the UI may be left in English, while the voiceover and cinematic assets are subtitled into Arabic. Such games include Ubisoft's Assassin's Creed Unity (2014) and Watch Dogs 2 (2016), which both lack an Arabic UI (Al-Batineh and Alawneh, 2021, p. 7). Other problematic localization practices include providing Arabic dubbing for the game's audio and cutscenes while leaving other game assets in English. The developers of Uncharted 4: A Thief's End (2016), Marvel's Spider-Man (2018), and Days Gone (2019) adopted this approach, which left important game assets (messages, instructions, settings, maps) inaccessible to Arab gamers due to the language barrier (Al-Batineh and Alawneh, 2021, p. 11). The unavailability of Arabic subtitles in localized video games also hinders playability for Arab gamers who are hearing-impaired. This issue occurs in Disney Infinity (2013), Forza Motorsport 6 (2015), World of Tanks Blitz (2016), and Lego Worlds (2017); the localizations of these games include Arabic re-voiced audio and cinematics without Arabic subtitles. Developers may be leaving these unresolved issues in the game due to time constraints, or technical and financial matters.

\subsubsection{Video game terms and acronyms in Arabic}

When translating games into Arabic, two more elements need to be considered by video game developers: the length of Arabic equivalent terms, especially video game menu terms, and the absence of acronyms in Arabic. The translation of interface items into Arabic takes more space as compared to English. Figures 8-A and 8-B show the "Home" button in the English and the Arabic versions of Captain Tsubasa: Rise of New Champions (2020). The Arabic equivalent "الصفحة الرئيسية" needs two lines and fifteen characters as compared to the one line and four characters in English.

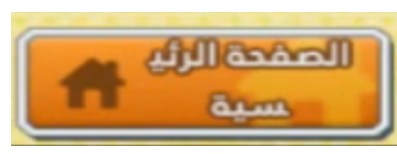

Figure 8-A: Arabic home button from Captain Tsubasa: Rise of New Champions (2020)

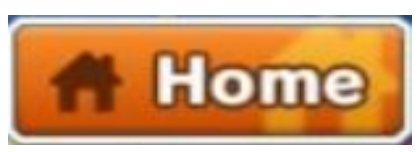

Figure 8-B: English home button from Captain Tsubasa: Rise of New Champions (2020)

The second Arabic word "الرئيسية" is spread over two lines because the game code does not allow menu item expansion. The ability to expand menu items should be taken into consideration when developing a game that will be localized into Arabic. In this regard, Chandler and Deming (2012) state: "[t]raditionally, the translated text is about $20 \%$ to $30 \%$ larger than sourcelanguage text, so if the UI is designed strictly for a specific language, the translated text in the UI will either be cut off or overlap in areas" (p. 5). Another 
example, found almost in all localized video games, is the Arabic translation of "hold" (four characters) as الضغط مطو لا على زر (fifteen characters).

\subsection{Cultural issues: Profanity, nudity, and alcohol}

Arab culture is very conservative in regard to sexuality, alcohol, and profanity. Moreover, topics that go against the cultural and religious beliefs of Arabs and Muslims are, in most cases, censored in audiovisual (AV) material by Arab audiovisual commissions that take such issues seriously. If the material cannot be censored, then the AV material can be banned altogether (Al-Adwan \& Yahiaoui, 2018). Several games have been banned in Arab countries for featuring content that subverts the cultural norms and traditions of Arab culture. Examples of video games banned in some parts of the Arab world include Assassin's Creed II (2009), Resident Evil 6 (2012), Grand Theft Auto V (2013), Deadpool (2013), and Mafia 3 (2016). Depending on the game, they were banned for violence and/or references to sexuality and sexual orientation (homosexuality), which are considered taboo in the Arab world.

Other games were rejected and banned by Arab gamers for including implicit or explicit insults to Muslims. Call of Duty: Modern Warfare 2 (2009) is a case in point. The game ignited rage in the Arab Muslim gaming community for insulting Islam because a short bathroom scene contained Arabic calligraphy of quotes from the prophet Mohammed. In response to the gamer community, the company has officially responded by apologizing and editing the scene in their update of the game. Another case was Resident Evil 5 (2009) in which an interactive scene allows the Holy Quran to be thrown on the floor and stepped on by the player; such a disrespectful scene enraged the Arab gaming community and forced the game developer to remove it in an update.

When it comes to localizing video games into Arabic, game companies should seriously consider such concerns. Their localized games should adhere to the cultural norms of the Arab world to avoid what Edwards (2012) calls "cultural dissonance" (p. 25), which will lead to a loss in video game sales and negatively impact the developer's image in some markets. In order to avoid cultural dissonance, the video game development team should consider localization into Arabic during the internationalization phase of the video game. This step would save them time and effort later, especially when a game contains sensitive content such as nudity and violence, and would save the game from being rejected by consumers or by censors.

\section{Conclusion}

The linguistic and technical issues discussed in the present paper negatively impact the playability of video games localized into Arabic, and consequently negatively affect the gaming experience of Arab players. The cultural issues, on the other hand, hinder the sales of such localized video games in the Arab countries, resulting in financial loss for the game developers and marketers.

Several issues in Arabic video game localization discussed above could be avoided and minimized by adopting a proper internationalization strategy. Additionally, video game development teams should include localizers or developers who are familiar with the challenges related to Arabic video game localization. A more comprehensive team would help prepare video games to accommodate an Arabic version that actually looks and feels Arabic. Video game localization should also be conceived in light of Gouadec's (2007) definition of localization as the adaptation of a product to the "particular physical, technical, linguistic, cultural, ethnic, religious, philosophical, commercial, marketing, etc. conditions and requirements of an audience or users belonging to a specific "locale"' (p. 37). In other words, the process of localizing video games should not only account for the linguistic transfer of a game's assets but also the cultural environment of the receiving locale, 
including an array of norms governing the (un)acceptability of localized foreign video games. However, this depends on the level of localization desired by the video game developer, which could be based on time constraints as well as localization ROI and target market value considerations.

Furthermore, collaboration between video game developers and Arabictranslation training programs should be established to build the next generation of video game localizers. Problematic issues in game localization are perpetuated by a lack of specialized video game developers who know the characteristics and peculiarities of the Arabic language. More specialized training programs or courses in Arabic video game localization would help fill that need. More professional Arabic localization would presumably result in greater product acceptance, increased popularity and thus increased sales, justifying the added expense and effort in game development.

Overall, more research in the area of Arabic video game localization is urgently needed considering the large and growing market demand for games with seamless user experience in Arabic. Research into Arabic video game localization should not only flag linguistic, cultural, and technical issues but also establish an area of research that would help practitioners and video game developers provide a unique gaming experience for Arab gamers and video game fans.

\section{References}

Al-Batineh, M., \& Alawneh, R. (2021). Current trends in localizing video games into Arabic: localization levels and gamers' preferences. Perspectives, 1-20. https://doi.org/10.1080/0907676X.2021.1926520

Al-Batineh, M., \& Alawneh, R. (in press). Translation hacking in Arabic video game localization: The history and current practices. Translation Spaces.

Abu Kishek, M. (2016). The Arabic localization of video games: An analysis of translation and localization practices of selected linguistic assets [Unpublished Master's thesis]. Irbid, Yarmouk University. Retrieved from http://thesis. mandumah.com/Record/228681

Al-Adwan, A. (2019). Mapping Arabic subtitling conventions: The case of Dubai One and MBC 2. In S. Faiq (Ed.), Arabic translation across discourses (pp. 63-78). London: Routledge. https://doi.org/10.4324/9781351063388-5

Al-Adwan, A., \& Yahiaoui, R. (2018). Comedy under fire: Subtitling two and a half men into Arabic. In I. Ranzato \& S. Zanotti (Eds.), Linguistic and cultural representation in audiovisual translation (pp. 85-100). London: Routledge. https://doi.org/10.4324/9781315268552-6

Al-Mazrooa, N. (2018). Arabic localisation: key case studies for translation studies [Unpublished Doctoral Dissertation]. Cardif, Cardiff University. Retrieved form: https://orca.cf.ac.uk/117575/1/2018almazrooanaphd.pdf

Aziz, Y. Y. (1983). Transliteration of English proper nouns into Arabic. Meta: Journal des Traducteurs, 28(1), 70. https://doi.org/10.7202/001931ar

Bernal-Merino, M. (2007). Challenges in the translation of video game. Tradumàtica: Traducció $i$ Tecnologies de La Informació $i$ La Comunicació, 5. http://www.fti.uab.es/tradumatica/revista/num5/articles/02/02.pdf

Bernal-Merino, M. (2015). Translation and localization in video games: Making entertainment software global. London: Routledge.

Bernal-Merino, M. (2020). Key concepts in game localisation quality. In L. Bogucki \& M. Deckert (Eds), The Palgrave handbook of audiovisual translation and media accessibility (pp. 297-314). Cham: Springer International Publishing. https://doi.org/10.1007/978-3-030-42105-2_15

Campbell, C. (2013). How Western games are being "culturalized" for Arabic countries. Polygon. https:/www.polygon.com/2013/11/30/5148520/how-western -games-are-being-culturalized-for-arabic-countries

Chandler, H., \& Deming, S. O. (2012). The game localization handbook (2 ${ }^{\text {nd }}$ ed.). Sudbury: Jones \& Bartlett Publishers.

Czech, D. (2013). Challenges in video game localization: An integrated perspective. 
Explorations: A Journal of Language and Literature, 1, 3-25.

Dunne, K. (2006). A Copernican revolution. In K. Dunne (Ed.), Perspectives on localization (pp. 1-11). Amsterdam/Philadelphia: John Benjamins.

Edwards, K. (2012). Culturalization of game content. In H. Chandler \& S. Deming (Eds.), The game localization handbook (pp. 19-34). Sudbury: Jones \& Bartlett Learning.

Gouadec, D. (2007). Translation as a profession (Vol. 73). Amsterdam/Philadelphia: John Benjamins. https://doi.org/10.1075/btl.73

Jiménez-Crespo, M. (2013). Translation and web localization. Oxon: Routledge. https://doi.org/10.4324/9780203520208

Jiménez-Crespo, M. (2019). Localization. In M. Baker \& G. Saldanha (Eds), The Routledge encyclopedia of translation studies ( $3^{\text {rd }}$ ed.), pp. 299-304). Oxon: Routledge. https://doi.org/10.4324/9781315678627-64

LISA. (2004). Localization industry primer ( $2^{\text {nd }}$ ed.). Romainmôtier: Localization Industry Standards Association.

Mahasneh, A. A., \& Abu Kishek, M. T. (2018). Arabic localization of video games "Tomb RaiderTM (2013)": A start or a failure. Lebende Sprachen, 63(1), 47-62. https://doi.org/10.1515/les-2018-0003

Mangiron, C. (2016). Games without borders: The cultural dimension of game localisation. Hermeneus, 18, 187-208.

Mangiron, C. (2018). Game on! Burning issues in game localisation. Journal of Audiovisual Translation, 1(1), 122-138.

Mangiron, C. (2021). Found in Translation: Evolving Approaches for the Localization of Japanese Video Games. Arts, 10(1), 9. https://doi.org/10.3390/arts10010009

O'Hagan, M., \& Chandler, H. (2016). Game localization research and translation studies. In Y. Gambier \& L. van Doorslaer (Eds.), Border crossings: Translation studies and other disciplines (pp. 309-330). Benjamins. https://doi.org/10.1075/ btl.126.15oha

O'Hagan, M., \& Mangiron, C. (2013). Game localization: Translating for the global digital entertainment industry. Amsterdam: John Benjamins Publishing Company. https://doi.org/10.1075/btl.106

Šiaučiūnè, V., \& Liubinienè, V. (2011). Video game localization: The analysis of ingame texts. Studies about Languages, 19, 46-55. https://doi.org/10.5755/ j01.sal.0.19.945

Vinay, J.-P., \& Darbelnet, J. (1995). Comparative stylistics of French and English: A methodology for translation (J. Sager \& M. Hamel (trans.); $1^{\text {st }}$ ed.). Amsterdam: John Benjamins.

\section{Gameography}

Assassin's Creed Unity (Ubisoft 2014)

Assassin's Creed II (Ubisoft 2009)

Assassin's Creed: Syndicate (Ubisoft 2015)

Assassin's Creed Odyssey (Ubisoft 2018)

Assassin's Creed Valhalla (Ubisoft 2020)

Call of Duty: Modern Warfare 2 (Activision 2009)

Captain Majed (BANDAI NAMCO 1995)

Captain Tsubasa II (Tecmo 1990)

Captain Tsubasa: Rise of New Champions (Bandai Namco Entertainment 2020)

Days Gone (Sony Interactive Entertainment 2019)

Deadpool (Activision 2013)

Disney Infinity (Disney Interactive Studios 2013)

FIFA 15 (EA Sports 2014)

FIFA 2018 (EA Sports 2017)

Forza Motorsport 6 (Microsoft Studios 2015)

Ghost of Tsushima (Sony Interactive Entertainment 2020)

Goat Simulator (Coffee Stain Studios 2015)

Grand Theft Auto VI (Rockstar Games 2013)

Lego Worlds (Warner Bros. Interactive Entertainment 2017) 
Mafia 3 (2K Games 2016)

Marvel's Spider-Man (Sony Computer Entertainment 2018)

PES 2018 (Konami Digital Entertainment Co. 2017)

Player Unknown's Battlegrounds (PUBG Corporation 2017)

Resident Evil 6 (Capcom 2012)

The Dragon (Ramar International 1995)

The Division 2 (2018)

The Witcher 3 (CD Projeckt 2015)

This is Football (Sony Interactive Entertainment 2005)

Tomb Raider (Square Enix 2013)

Uncharted 4: A Thief's End (Sony Interactive Entertainment 2016)

Watch Dogs 2 (Ubisoft 2016)

World of Tanks Blitz (Wargaming Group Limited 2016)

Maquette (Graceful Decay 2021)

\section{Appendix 1: Analysed video games}

\begin{tabular}{|rl|}
\hline 1. & Assassin's Creed Unity (2014) \\
\hline 2. & Assassin's Creed II (2009) \\
\hline 3. & Assassin's Creed Valhalla (2020) \\
\hline 4. & Assassin's Creed Odyssey (2018) \\
\hline 5. & Call of Duty: Modern Warfare 2 (2009) \\
\hline 6. & Captain Tsubasa: Rise of New Champions (2020) \\
\hline 7. & Days Gone (2019) \\
\hline 8. & Deadpool (2013) \\
\hline 9. & Disney Infinity (2013) \\
\hline 10. & FIFA 2018 (2017) \\
\hline 11. & Forza 6 (2015) \\
\hline 12. & Ghost of Tsushima (2020) \\
\hline 13. & Goat Simulator (2015). \\
\hline 14. & Grand Theft Auto V I (2013) \\
\hline 15. & LEGO Super-Villains (2018) \\
\hline 16. & Lego Worlds (2017) \\
\hline 17. & Mafia 3 (2016) \\
\hline 18. & Maquette (2021) \\
\hline 19. & Marvel's Spider-Man (2018) \\
\hline 20. & PES 2018 (2017) \\
\hline 21. & PlayerUnknown's Battlegrounds (2017) \\
\hline 22. & Resident Evil 5 (2009) \\
\hline 23. & Resident Evil 6 (2012) \\
\hline 24. & The Division 2 (2018) \\
\hline 25. & The Witcher 3 (2015) \\
\hline 26. & Uncharted 4: A Thief's End (2016) \\
\hline 27. & Watch Dogs 2 (2016) \\
\hline 28. & World of Tanks Blitz (2016) \\
\hline & \\
\hline
\end{tabular}

